\title{
Ultrafast moving-spot microscopy: Birth and growth of laser-induced periodic surface structures
}

\author{
Mario Garcia-Lechuga,* Daniel Puerto, Yasser Fuentes-Edfuf, Javier Solis, and Jan Siegel* \\ Laser Processing Group, Instituto de Optica, CSIC, Serrano 121, 28006 Madrid, Spain \\ *corresponding authors: mario@io.cfmac.csic.es, j.siegel@,csic.es
}

\begin{abstract}
Laser-induced periodic surface structures (LIPSS) are a universal phenomenon observed in all classes of solid materials, giving rise to a variety of self-assembled subwavelength structures with different symmetries. These promising features have opened new opportunities for laser structuring of materials in a wide range of applications, including plasmonics, nanophotonics, nanoelectronics, sensing and even mechanics. However, there is an ongoing debate about the formation mechanism of LIPSS and the current picture stems mainly from the combined effort of theoretical modeling and experimental studies of the final structures produced. Here we demonstrate femtosecondresolved imaging of the formation process of such structures produced by ultrashort laser pulses in silicon. The particular type of LIPSS studied are well-aligned amorphouscrystalline fringes generated in dynamic processing conditions, whose period can be tuned and which can be extended over large areas. Using a moving-spot, multiple-pulse irradiation approach we are able to spatially and temporally resolve the birth and growth of individual fringes. We demonstrate that the formation process is initiated by free electron generation leading to non-thermal melting, liquid phase overheating and rapid solidification into the amorphous phase.
\end{abstract}

Keywords: femtosecond laser, time-resolved microscopy, laser induced periodic surface structures, ultrafast melting, phase change, silicon photonics

Nanostructuring material surfaces with novel textures and high precision has become a key requirement for scientific and technological progress in the vast field of nanotechnology. Amongst the numerous strategies employed, top-down approaches based on sculpting an initially featureless material are generally most established and have reached a high level of maturity. Prominent examples are lithographic techniques [1], in which complex patterns are designed and imprinted by photons, electrons, or ions. Bottom-up approaches consist in brick-like building of extended nanostructures by assembling atomic or molecular units [2], which would be prohibitively timeconsuming if of not performed in parallel. Self-assembly is arguably the perfect bottomup approach since an organized structure spontaneously forms as a result of local interactions among the components [3]. In practice though, structures fabricated by selfassembly processes tend to suffer from defects. This limitation has triggered new approaches based on guiding self-assembly mechanisms by external fields, leading to improved structures [4].

A highly flexible strategy of guided self-assembly is based on ultrafast laser irradiation of material surfaces, leading to the formation of so-called Laser-Induced Periodic Surface Structures (LIPSS) [5,6,7,8,9,10,11]. The incident laser light interferes with a scattered surface wave or excited surface plasmon polarition (SPP) [12], leading

ACS Photonics, 2016, 3 (10), pp 1961-1967, DOI: 10.1021/acsphotonics.6b00514 Publication Date (Web): September 16, 2016, Copyright (C) 2016 American Chemical Society 
to a modulated intensity distribution, which is the driving force for the self-organization process. Different models have been developed to relate material parameters to the specific LIPSS features produced [11,12,13,14,15,16,17]. While most works attribute LIPSS formation to the presence of SPPs, Zhang et al. have shown recently by means of modeling that near- and far-field scatter fields interfering with direct laser light have the potential to trigger formation of low and high spatial frequency LIPSS structures [17]. Experimentally, high-quality structures based on this process have been produced by exploiting local and non-local feedback mechanisms [10]. Despite the enormous progress made in this field, fundamental questions about the underlying mechanisms and their dynamics remain open. Höhm et al. [18] have used an indirect, though powerful method to extract information about the formation dynamics by performing double pump pulse experiments and analyzing the final structure as a function of the pulse delay. But only very few experimental works exist $[19,20,21]$ that attempt to directly monitor the formation dynamics of LIPSS, which is essentially due to the multiple challenges posed to the experimental technique employed. Sokolowski-Tinten and co-workers [19] have addressed some of these challenges by means of single-shot pump-probe coherent XUV-scattering techniques using a free-electron-laser. The authors observed indications of a periodic structure that started to form during the picosecond pulse and lasted up to 2 ns, after which the sample started to disintegrate due to the high excitation level. Kafka et al [20] employed a pump-probe optical microscopy technique detecting scattered light upon the formation of LIPSS in Cu. In order to facilitate LIPSS formation upon a single femtosecond pulse they used a custom-made sample with a mechanically formed groove. Jia et al. [21] employed an optical time-resolved imaging technique with a temporal resolution of $1 \mathrm{ps}$ and a spatial resolution of $440 \mathrm{~nm}$ to study LIPSS formation in Si. They observed an onset of periodic ripples formation tens of picoseconds after material excitation and found the formation process to end after $1.5 \mathrm{~ns}$.

While providing some insight into the underlying mechanism, all strategies are limited to static irradiation with a maximum lateral LIPSS extension of a few tens of micrometers. Furthermore, they fail to convincingly address the issue of pulse number, which is crucial for LIPSS as the final structure depends on the number of laser pulses. The technique presented in this paper has the ability to acquire the pulse number dependence in parallel, inherently disentangling its inter-relation with the laser fluence. The strategy allows directly resolving and monitoring the LIPSS formation process with fs temporal and submicron lateral resolution in true writing conditions of laterally extended grating structures. In a recent work we have shown that lines and areas with parallel LIPSS patterns formed of amorphous and crystalline fringes can be written in silicon by careful balance of pulse energy, laser repetition rate and scan speed [22]. Translating these conditions to a stepwise moving-spot excitation approach and synchronizing it with optical pump-probe microscopy [23,24,25] allows us to unravel the dynamics of the LIPSS formation process.

\section{Results and discussion}

Fig. 1 shows the principle of the pump-probe microscopy technique (see also Methods): An ultrashort pump pulse incident at an angle on the sample surface triggers LIPSS formation while a delayed ultrashort probe pulse is used for illumination. The illuminated area is imaged by means of a microscope objective onto a charge-coupled device camera (CCD). The delay between pump and probe pulses can be adjusted in

ACS Photonics, 2016, 3 (10), pp 1961-1967, DOI: 10.1021/acsphotonics.6b00514 
steps of $50 \mathrm{fs}$. We have chosen a strategy to move the sample by an amount equal to the fringe period between consecutive pulses, which serves a double purpose. First, it ensures that extended lines and areas of amorphous-crystalline fringes are written by providing the optimum pulse number for maximum fringe contrast [22]. Second, it ensures that each excitation pulse is incident onto an equivalent fringe structure at the same spatial position.
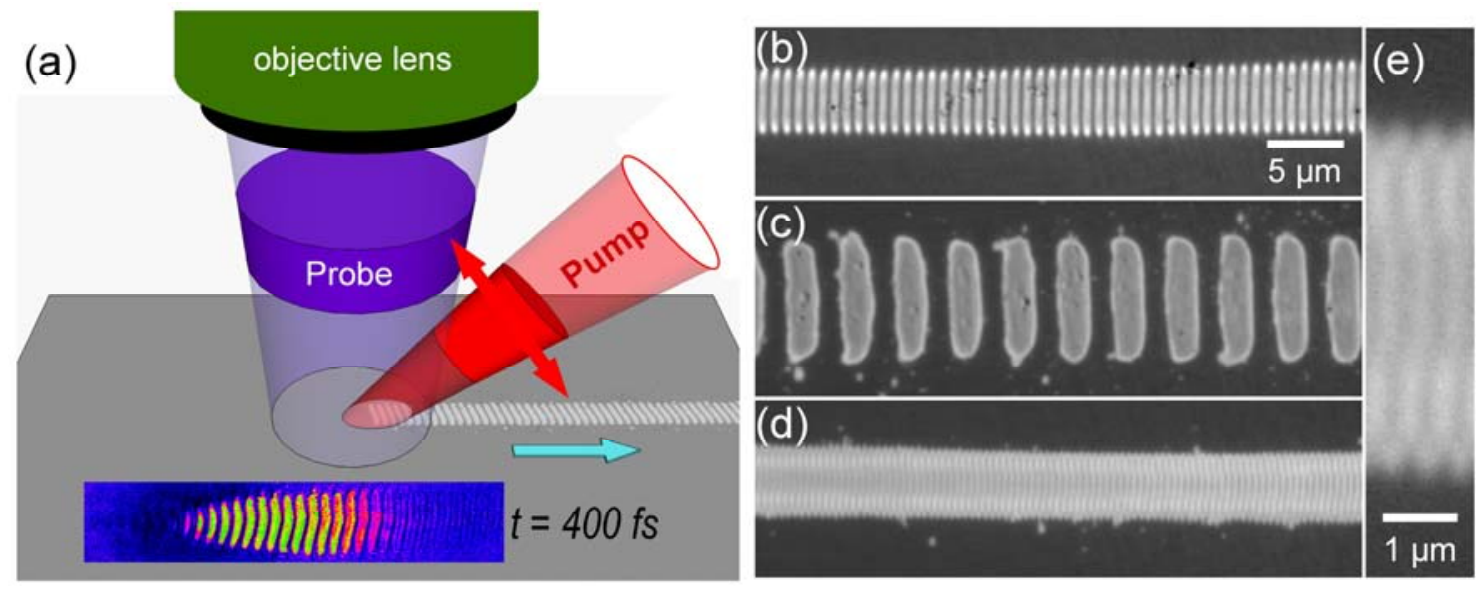

Fig. 1: (a) Sketch of the ultrafast moving-spot microscopy technique. The p-polarized (red arrow) femtosecond pump pulse excites the Si sample and triggers LIPSS formation. The sample is moved between excitation pulses in steps equal to the fringe spacing along the direction of the cyan arrow. A femtosecond probe pulse is sent through a microscope objective to illuminate the structure at a userselectable delay. The inset shows an image of the transient surface reflectivity change recorded at a pump-probe delay of $t=400 \mathrm{fs}$. (b-d) Optical micrographs of amorphous-crystalline nanofringe lines written at (b) normal beam incidence or (c,d) angular incidence of $52^{\circ}$. For (d) the sample was moved in the opposite direction. (e) Zoomed region of (d)

The regularity and high contrast of the amorphous-crystalline fringes formed by the pump beam is illustrated in Fig. 1(b-d). Depending on the angle of incidence and writing direction, very different periods can be obtained for a single wavelength, namely $\Lambda_{b\left(0^{\circ}\right)} \approx 780 \mathrm{~nm}, \Lambda_{c\left(52^{\circ}\right)} \approx 3.53 \mu \mathrm{m}$ and $\Lambda_{d\left(52^{\circ}\right)} \approx 440 \mathrm{~nm}$. An angle dependence of LIPSS in static irradiation experiments has been observed earlier [8], reporting two possible periods for p-polarized light, according to $\Lambda^{s-}=\lambda /(1+\sin \theta)$ and $\Lambda^{s+}=\lambda /(1-\sin \theta)$, which were found to co-exist in form of a superposition both periods. Our results demonstrate that this coexistence can be overcome by a proper selection of the movement direction, strongly enhancing one period and suppressing the other. Whether this simple but effective strategy might also work for standard ablative LIPSS remains to be explored, we are not aware of related studies.

Fig. 2 shows images recorded with the moving-spot pump-probe microscope during the writing process. Fig. 2(a) features an image of the front of the fringe line being written, in which the upper half-image has been recorded before exposure to a further pump pulse, whereas the lower part shows the resulting changed ripple front $1 \mathrm{~s}$ after the pump pulse. By comparing both sub-images, an increase in contrast and lateral extension of a few fringes near the front, as well as the formation of a new, short fringe with weak contrast at the very front can be observed. The temporal evolution from the "before"-state to the "after"-state can be followed in the sequence of four time-resolved in-situ microscopy images shown in Fig. 2(b-e). As opposed to Fig. 2(a) these images have been normalized with respect to the "before"-state and show the relative transient change of reflectivity of the structure induced by single pulse (see also Methods). 

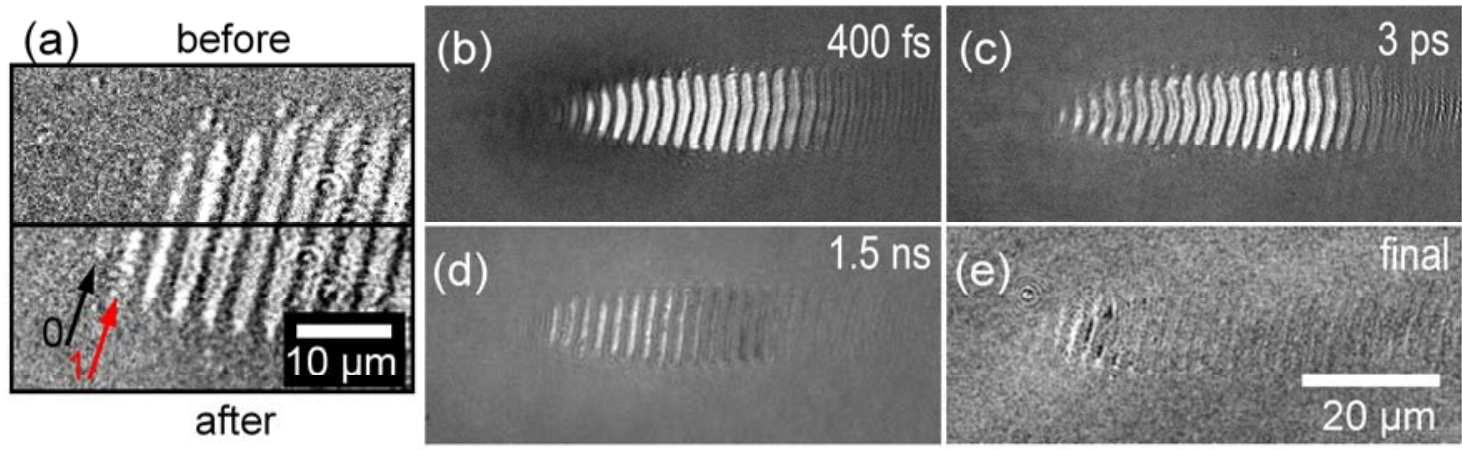

Fig. 2: In-situ microscopy images of the Si surface during laser writing of amorphous fringes. (a) The upper half section of the frame corresponds to a micrograph of the fringes before the arrival of an excitation pulse, the lower section to one afterwards, at a long delay (1 s). The arrow labeled 1 points to a fringe that is weakly present before and whose contrast is increased after irradiation, whereas fringe 0 is newly formed by the pulse. (b-e) Series of images recorded at different delay times (c.f. image labels) after arrival of the excitation pulse. The images in $(b-e)$ display relative reflectivity changes with respect to the fringe structure before irradiation.

The formation dynamics of these ripples can be followed by recording images at ultrashort delays. At a pump-probe delay of $400 \mathrm{fs}$, a pronounced change in reflectivity can be appreciated at the leading edge of the fringe front in Fig. 2(b). This strong reflectivity increase lasts hundreds of picoseconds before the reflectivity begins to decrease in the nanosecond delay range (c.f. $t=1.5$ ns, Fig. 2(d)). The difference in the overall shape of the ripple front between images is caused by the fact that each image corresponds to a different region (moving spot approach) and that surface imperfections influence fringe quality. It is worth noting that the size of the excitation spot (horizontally elliptic with $1 / \mathrm{e}^{2}$-diameters of $170 \mu \mathrm{m} \times 100 \mu \mathrm{m}$ ) is much larger than the region in which transient changes of the fringe structure can be observed, which is due to the low fluence for stable fringe formation $\left.\left(F_{\text {fringe }}=140 \mathrm{~mJ} / \mathrm{cm}^{2}\right)\right)$.

For a quantitative analysis of the transient reflectivity changes we have build a composite image (Fig. 3), composed of horizontal slices of transient images shown in Fig. 2 forming the spatial dimension, stacked vertically for different delays that form the temporal dimension, with the reflectivity change being false color-coded as the third dimension (see Methods). It is worth emphasizing that the spatial dimension along the horizontal axis provides simultaneously a fourth dimension, the effective pulse dose $N_{\text {eff }}$ per fringe. This fact is due to the moving-spot approach of the technique, in which the sample is moved between pulses in steps equal to the fringe spacing $\left(\Lambda_{c\left(52^{\circ}\right)} \approx 3.53 \mu \mathrm{m}\right)$ to the right, leading to an increase in pulse number to the right.

In order to ease interpretation of this complex map we have performed a separate experiment, investigating the temporal evolution of the reflectivity of a static and unexposed Si surface upon single pulse irradiation, not generating LIPSS. In this way the specific features of the ultrafast response of Si can be discussed and used as a benchmark for the analysis of the fringe formation dynamics, being significantly more complex. Sokolowski-Tinten et al. have studied the material response of Si upon ultrashort laser excitation below the ablation threshold, employing somehow different irradiation parameters and point-probing [26,27]. The authors identified several key stages, which we do observe nicely in our images and use as a reference for the interpreting the data obtained on fringe formation employing the moving-spot technique, as discussed in the following sections. 
In Fig. 3 (right column, single pulse data) a slight reflectivity increase surrounded by an annular decrease can be observed at a delay of 150 fs. This ultrafast optical response can be explained by free carrier generation due to the high laser peak intensities involved. Using a simple Drude model (see Methods) we have calculated the evolution of the surface reflectivity of c-Si at the illumination wavelength as a function of free electron density $n_{e}$ for our experimental conditions. The data obtained (c.f. pink curve in Fig. 4(c)) features an initial decrease of reflectivity before increasing above the level of the non-excited material at the critical electron density. A comparison of this curve to the single pulse data allows an estimation of the transient electron densities reached at the spot center $\left(n_{e}=4 \times 10^{22} \mathrm{~cm}^{-3}\right)$ and the outer ring of decreased reflectivity $\left(n_{e}=1 \times 10^{22} \mathrm{~cm}^{-3}\right)$.

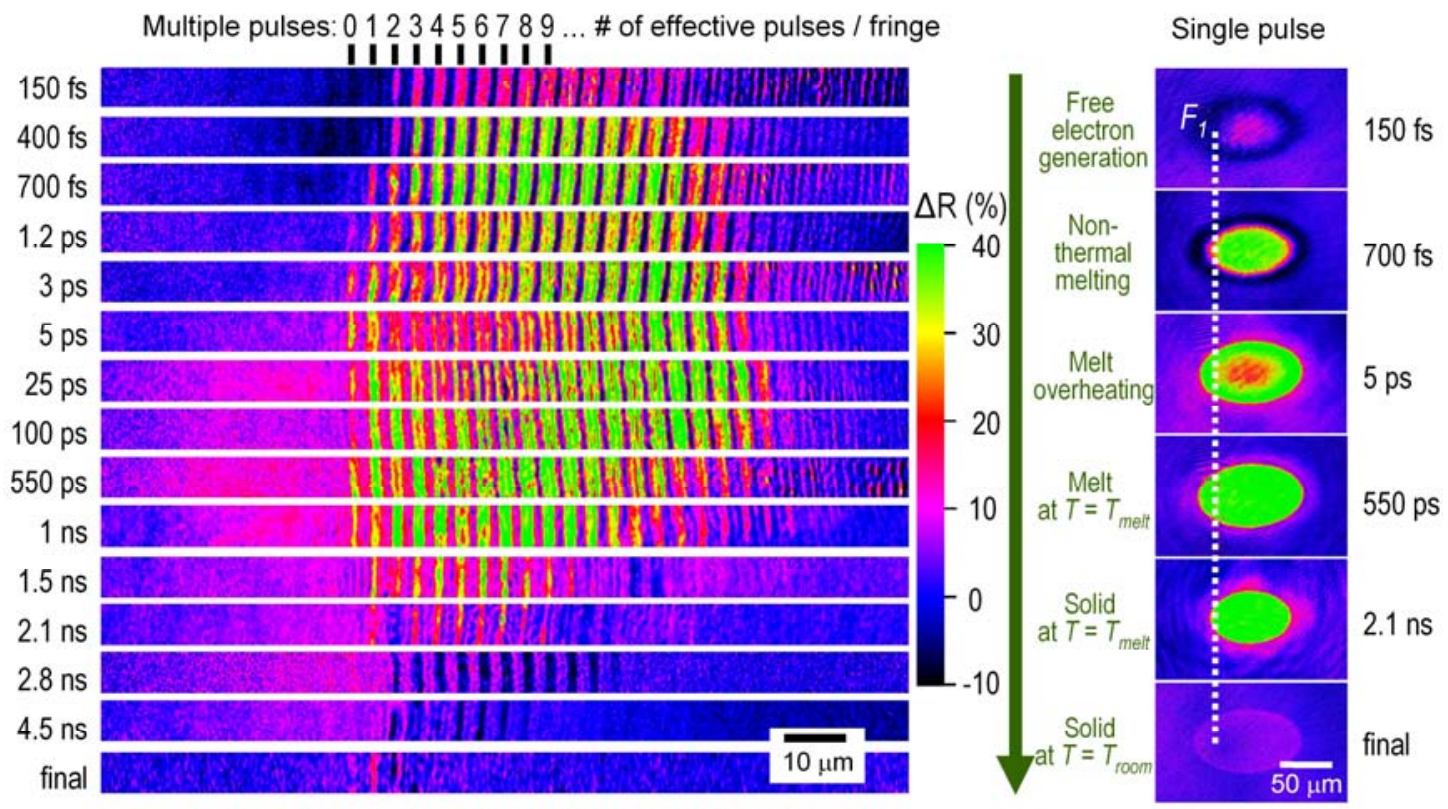

Fig. 3: (Left) Temporal sequence of horizontal slices of time-resolved images like those shown in Fig. 2, using a false-color scale for the reflectivity change $\Delta R$. Since the data has been obtained in a moving-spot configuration, each fringe has received a different effective number \# of pulses, increasing from left to right. Fringe \#0 (pulse dose 0) corresponds to the fringe being formed by the current excitation pulse. Fringe \#1 has already been exposed to a previous excitation pulse, etc. (Right) Selected time-resolved images of single pulse excitation of non-structured $c$-Si for comparison. $F_{1}$ marks the lateral position corresponding to the local fluence $F_{1}$ evaluated in Fig. 4. (Middle) The vertical time axis lists the sequence of key stages of the material response to irradiation with a single laser pulse, which can also be identified in the fringe response upon multiple pulse irradiations.

When comparing the single pulse data to the data on fringe formation at the same delay, care needs to be taken to limit the comparison to crystalline regions. These are either those between amorphous fringes or fringe \#0, which is being formed by the very laser pulse used to record the image. A reflectivity profile of the image at $t=150 \mathrm{fs}$ is displayed in Fig. 4(a). While for fringe \#0 and \#1 and near to them negative $\Delta R$ values around $-5 \%$ are obtained at this delay, fringe \#2 shows a increase of $10 \%$, comparable to the minimum and maximum values obtained in the single pulse data at this delay (c.f. Fig. 3, right). An important conclusion that can be drawn at this point is that the maximum transient electron density achieved during fringe formation lies in the range $n_{e}=1-4 \times 10^{22} \mathrm{~cm}^{-3}$. This experimentally determined $n_{e}$-range is considerably higher than the one predicted from modeling for ablative LIPSS in Si $\left(n_{e}=1-7 \times 10^{21}\right.$ $\mathrm{cm}^{-3}$ ) [9], yet consistent with experimental results for single-pulse ultrafast melting [27].

The data at ultrashort time delays also provides information on the possible presence of surface plasmon polaritons (SPPs) $[13,14]$. On the one hand, our results

ACS Photonics, 2016, 3 (10), pp 1961-1967, DOI: 10.1021/acsphotonics.6b00514 Publication Date (Web): September 16, 2016, Copyright (C) 2016 American Chemical Society 
show that the free electron density of c-Si is strongly increased by the laser, approaching that of metals for which SPPs can potentially be observed. On the other hand, if present, one would expect a modulation in reflectivity in form of dark fringes reaching out far to the left of fringe \#1. In fact, the profile at $t=150 \mathrm{fs}$ in Fig. 4(a) suggests such a weak periodic modulation. The expected strong attenuation of a SPP wavepacket in absorbing materials might explain that only a few periods can be observed. Yet, attenuation in Si, even when excited, should be considerably less than in metals. Alternatively, the excitation pattern we observe, confined near the fringe front, is fully consistent with being triggered by far- and near-field scattering, interfering with the directly incident laser light as proposed in [17], which also explains the period observed experimentally.

For an estimation of $n_{e}$-values within already well-formed (amorphous) fringes (\#2 and higher), the cyan curve in Fig. 4(c) needs to be considered, corresponding to the calculation for amorphous $\mathrm{Si}$. The predicted absence of negative $\Delta R$-values is indeed observed experimentally. The positive $\Delta R$-increase is larger, pointing towards higher $n_{e^{-}}$ values than in the c-Si regions. This stronger excitation is consistent with the high linear absorption coefficient of the amorphous phase $\left(k_{a-S i}=0.109\right.$ compared to $k_{c-S i}=0.006$ at $800 \mathrm{~nm}$ ) dominating over the combined action of linear and two-photon absorption in the crystalline phase [27].
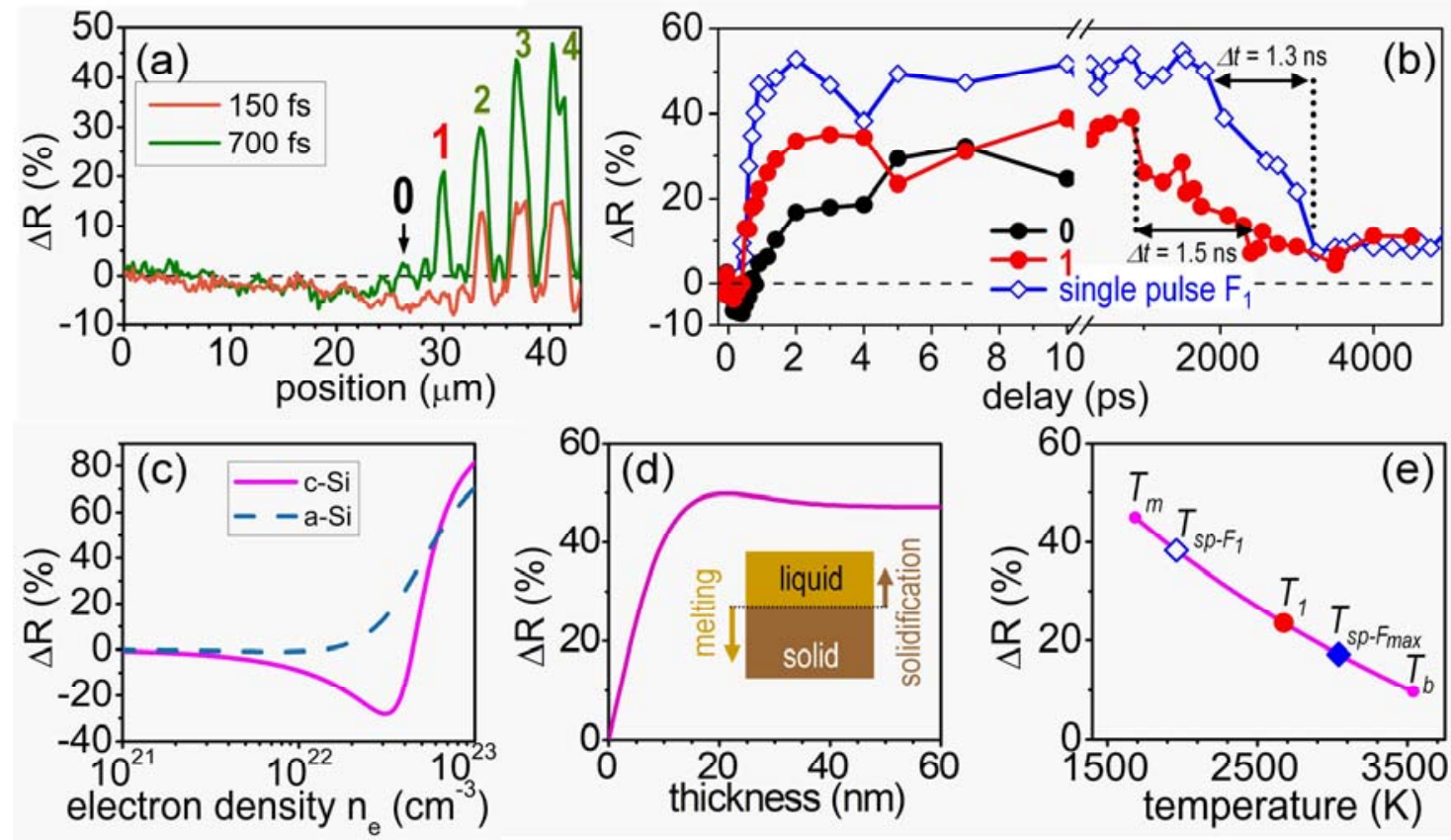

Fig. 4: (a) Horizontal cross sections of the transient reflectivity map in Fig. 3 at delays $t=150 f_{s}$ and 700

$f_{s}$. The labels refer to the fringe \#. (b) ) Temporal evolution of the reflectivity change of the center of fringes \#0 (black curve) and \#1 (red curve) extracted from Fig. 3 by means of vertical profiles. The blue curve shows the corresponding evolution of non-structured Si upon single pulse excitation at fluence $F_{1}$ for comparison. (c) Calculated surface reflectivity change of crystalline (pink curve) and amorphous $\mathrm{Si}$ (cyan curve) as a function the electron density using a Drude model. (d) Calculated reflectivity change of crystalline Si upon melting, i.e. formation of a liquid top layer, starting at the surface. The reflectivity response upon solidification from the melt can be obtained by following the curve from right to left. (e) Reflectivity change of molten Si as a function of temperature. The curve shows the calculated data from the melting point $\left(T_{m}\right)$ up to the boiling point $\left(T_{b}\right)$, using a Drude model. The data points correspond to the experimental reflectivity minima in the delay range of 2 ps -10 ps in Fig. 4(b) of fringe \#1 ( $\left.T_{1}\right)$, single pulse at $F_{1}\left(T_{s p-F l}\right)$ and single pulse at the peak fluence $\left(T_{s p-F m a x}\right)$. 
Following the excitation stage, the material melts at sufficiently high pulse fluence. The molten phase of Si is characterized by metal-like state, featuring a high reflectivity in the visible region. The optical properties of molten Si are known [28] and can be used to calculate the expected reflectivity increase upon surface melting as a function of melt depth (c.f. Fig 4(d), see also Methods). This first-order phase transition may occur through different pathways and with different time scales, depending on the fluence used. The blue curve in Fig. 4(b) shows the reflectivity evolution of the single pulse experiment at a fluence $F_{1}=170 \mathrm{~mJ} / \mathrm{cm}^{2}$, above the melting threshold $\left(F_{m}=134\right.$ $\mathrm{mJ} / \mathrm{cm}^{2}$, c.f. Methods for its determination). The ultrafast reflectivity rise within $\Delta t=$ $600 \mathrm{fs}$ is consistent with non-thermal melting due to a lattice instability induced by a high density of free electrons [26,29]. This conclusion is in agreement with our estimated $n_{e}$-values, which exceed the threshold for non-thermal melting $\left(n_{e, t h r e s h} \approx 10^{22}\right.$ $\left.\mathrm{cm}^{-3}\right)$ [27].

An equally fast reflectivity rise is observed for fringe \#1 (red curve in Fig. 4(b)), indicating non-thermal melting. It is worth noting that the slightly lower reflectivity maximum is not an indication of a shallow melt depth but a result of the narrow fringe width $(\approx 1.8 \mu \mathrm{m})$, leading to an averaging effect of different local fluences caused by the limited spatial resolution of our microscope. A slower reflectivity rise is observed for fringe \#0 (black curve in Fig. 4(b)) obtaining its maximum reflectivity value at $t=7$ ps, pointing towards thermal melting, induced by electron-phonon coupling [26]. The different transition times can be understood by the stronger excitation of \#1, being partially amorphous before excitation and having thus a higher linear absorption coefficient. Another important conclusion that can be drawn in this context is that the crystalline regions between amorphous fringes have not experienced melting and rapid solidification, despite the free electron plasma generated in this region.

An interesting feature of the reflectivity response of both, bare $\mathrm{Si}$ and fringes can be observed at delays around $t=5$ ps (Figs. 3 and 4), featuring a notable decrease. Yet, this behaviour is not a sign of surface ablation, since no crater is formed afterwards and the reflectivity of the molten phase recovers at longer delays. This transient reflectivity decrease is caused by the high temperature of the liquid, behaving similar as a metal upon heating. Several groups have studied this behavior using a double pulse excitation configuration, in which the second pulse heats the melt [30,31]. A modified Drude model (see also Methods) can be used to describe the reflectivity change upon heating. Fig. 4(e) shows the resulting curve from the melting point $\left(T_{m}\right)$ up to the boiling point $\left(T_{b}\right)$. By placing the values of the reflectivity dips of the experimental data in Fig. 4(b) on this curve, an estimation of the transient maximum overheating of the melt can be done. This way, an overheating of $\Delta T=280 \mathrm{~K}$ above $T_{m}$ is found for the single pulse experiment at $F_{1}$ and $\Delta T=990 \mathrm{~K}$ for fringe \#1. Even higher values are found for the single pulse experiment at the peak fluence $\left(\Delta T=1360 \mathrm{~K}\right.$ at $\left.F_{\max }=240 \mathrm{~mJ} / \mathrm{cm}^{2}\right)$, yet still being below the boiling point, which is consistent with the absence of an ablation crater.

Upon cooling of the melt at longer delays, solidification takes place. In bulk c$\mathrm{Si}$, this process is known to occur interfacially with velocities $v_{\text {int }}$ depending mainly on irradiation parameters. For Si $<100>$ Thompson reported epitaxial recrystallization to take place for $v_{\text {int }}<13 \mathrm{~m} / \mathrm{s}$ and a shallow melt depth, whereas amorphization is observed for higher velocities [32]. An estimation of $v_{\text {int }}$ in our experimental conditions can be made from Fig. 4(b), by measuring the decay time (90\% to $10 \%$ criterion) from the high reflectivity value (molten phase) to the value of the solid phase at the melting temperature (see Methods) [33]. According to Fig. 4(d), this solidification time

ACS Photonics, 2016, 3 (10), pp 1961-1967, DOI: 10.1021/acsphotonics.6b00514

Publication Date (Web): September 16, 2016, Copyright (C) 2016 American Chemical Society 
corresponds to propagation of the liquid-solid interface from a depth of $d=20 \mathrm{~nm}$ to the surface. For the single pulse experiment this occurs in $\Delta t \approx 1.3 \mathrm{~ns}$, leading to $v_{\text {int }}=\mathrm{d} / \Delta t$ $\approx 15 \mathrm{~m} / \mathrm{s}$, above the threshold for amorphization, which is consistent with the partially amorphous state after irradiation. For fringe \#1, a solidification time $\Delta t \approx 1.5$ ns and $v_{\text {int }}$ $\approx 13 \mathrm{~m} / \mathrm{s}$ can be estimated; just above threshold and consistent with the partial amorphous state after solidification.

\section{Conclusions}

We report an optical method capable of simultaneously generating laser-induced self-assembled periodic structures and resolving spatially and temporally their formation process. The method benefits from a moving spot-approach, which ensures the formation of high quality structures and constant irradiation conditions, allowing the parallel acquisition of the formation dynamics for different pulse doses. Applied to the case of periodic amorphous-crystalline patterns in silicon, we have investigated the dynamics of the formation process, identifying different key stages and their corresponding time scales, starting with the generation of free electrons at high density, and leading to non-thermal melting, liquid phase overheating and rapid solidification into the amorphous phase. The results are consistent with an excitation process being triggered by far- and near-field scattered light that interferes with the directly incident laser light, without need to involve surface plasmon polaritons. Our technique has the potential to clarify the formation dynamics and mechanisms of other laser-induced periodic surface structures, whose origin is still under debate.

\section{Methods}

\section{Moving-spot femtosecond microscope}

The laser system used is a Ti:sapphire femtosecond amplifier providing pulses of 120 fs FWHM (Full Width at Half Maximum) at $800 \mathrm{~nm}$ center wavelength with a repetition rate of $100 \mathrm{~Hz}$. A mechanical shutter selects a single pulse, which is split into two fractions: pump and probe. The pump pulse is p-polarized and focused at the sample surface at an angle of $52^{\circ}$ to generate and excited elliptical region with a

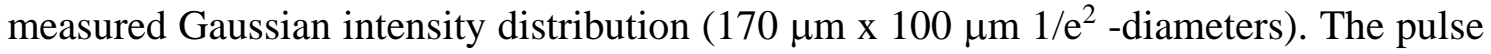
energy is controlled by a combination of half wave plate and polarizing cube beamsplitter. The probe pulse is frequency-doubled to $400 \mathrm{~nm}$ and used to illuminate the sample surface through an objective lens at normal incidence. The same objective $(20 \mathrm{x}$, N.A. $=0.42)$ collects the probe light and projects in combination with a tube lens an enlarge image of the sample surface onto a 16 bit CCD camera. For performing moving spot microscopy of LIPSS, the first stage is to generate a situation of stable LIPSS formation upon moving the spot over the sample surface. This is done by initially exposing the static sample to an elevated number of pulses at the optimum fluence for LIPSS formation. This leads to amorphization of a large spot, accompanied by minor surface damage. Then, the sample is moved in discrete steps equal to the fringe period $(\Lambda=3.53 \mu \mathrm{m})$ and performing single pulse exposure after each movement, which leads to the progressive formation of fringes. After about 30-50 steps the fringe line is fully developed and the fringes are parallel and well-.aligned. This is the starting condition for the time-resolved study, which consists in moving the sample one step, recording an image illuminated with a probe pulse without excitation ("before"), with excitation ("pump-probe”), and without excitation ("after"). Afterwards the sample is

ACS Photonics, 2016, 3 (10), pp 1961-1967, DOI: 10.1021/acsphotonics.6b00514

Publication Date (Web): September 16, 2016, Copyright (C) 2016 American Chemical Society 
moved a further step and the sequence is repeated for a different probe delay. The acquisition procedure is illustrated schematically in Fig. 5. It should be emphasized that all images displayed and analyzed in this paper are single shot images. The reproducibility is very high, which has been tested by recording several images at constant delay.

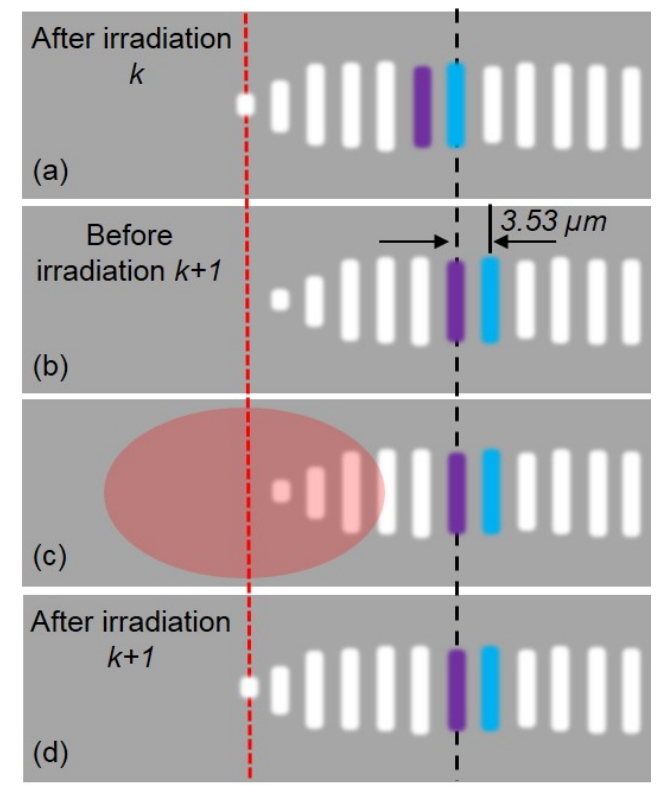

Fig. 5: Scheme of the image acquisition procedure, illustrating the field of view seen by the recording $C C D$ camera. Fringes are displayed as white vertical bars with two fringes being colored purple and blue in order to track their positions upon sample movement (from left to right). The red vertical line marks the horizontal center axis of the irradiation spot (red ellipse in (c)), the black vertical line is employed to reference the initial position of the blue fringe. (a) Fringe distribution after the previous irradiation $k$. (b) Fringe distribution after moving the sample to the right by one fringe period, corresponding to the "before" image recorded. (c) Same fringe distribution as in (b) being exposed to a single laser pulse ( $k+1)$, corresponding to the "pump-probe" image recorded. (d) New fringe distribution formed by the excitation pulse performed in (c), leading to the generation of a new fringe along the red line and to a vertical extension of neighbor fringes. This distribution corresponds to the "after" image recorded.

\section{Image analysis}

Image normalization is performed by dividing for each delay the corresponding "pump-probe" image by a "before" image and the "after" image by the "before" image, saving the resulting normalized images in 32bit format. The values are then converted into reflectivity percentage changes by subtracting 1 and multiplying by 100. From the treated image horizontal cross sections of 20 pixel height are cropped and color-coded to fabricate Fig. 3. Care was taken to position the cross sections at the correct horizontal position, which was done by aligning first the "after" images for each delay. The temporal evoluation of fringes shown in Fig. 4(b) was obtained by narrowing down the 20 pixel high horizontal profiles to stripes of 5 pixel height, performing a precise horizontal alignment of all cross sections to achieve fringe continuity along different delay times and finally plotting the central pixel of each fringe as a function of delay time.

\section{Definition of melting threshold and solidification time}

Following the criterion given in [27], we define the melting threshold as the lowest pump fluence for which the observed reflectivity rise equals the reflectivity $R_{\text {solid }}\left(T_{m}\right)$ of solid silicon at the equilibrium melting temperature of $1685^{\circ} \mathrm{K}$. A simple

ACS Photonics, 2016, 3 (10), pp 1961-1967, DOI: 10.1021/acsphotonics.6b00514 
method to determine $R_{\text {solid }}\left(T_{m}\right)$ with good precision is by taking the reflectivity level immediately after solidification, e.g. In Fig. 4(b) for the single pulse experiment at $t>$ $3.5 \mathrm{~ns}$, yielding $\Delta R_{\text {solid }}\left(T_{m}\right)=8 \%$.

\section{Modeling}

A Drude model was used to calculate the contribution of the photo-generated free electrons (ne) to the dielectric function of the laser-excited Si for Fig. 4(c). Details are described in [34]. For Si we used $m^{*}=0.18$ and $t_{d}=1 \mathrm{fs}$ as values for the optical mass and damping time, respectively, following the argument in Ref. 22.

The calculation of the reflectivity change upon melting shown in Fig. 4(d) was performed with a program relying on on an exact mathematical description of the interaction of an electromagnetic wave with an isotropic planar multilayered system formed by layers with different refractive indexes and absorption coefficients. The program transforms layer by layer in steps of $1 \mathrm{~nm}$ the optical constants of c-Si into those of liquid $\mathrm{Si}$ and calculates the reflectivity of the whole system at the probe wavelength $(400 \mathrm{~nm})$ as a function of the transformation depth.

Furthermore, the Drude model mentioned above was modified in order to describe the reflectivity behavior upon heating of molten Si. In a first approximation, the plasma frequency is assumed constant and the collision frequency $\omega_{c}$ to increase linearly with temperature according to $\omega_{c}(T)=\omega_{c}\left(T_{m}\right) \cdot T / T_{m}$, with $T_{m}$ being the melting point $(1685 \mathrm{~K})$ [30]. We have calculated the reflectivity change as a function of temperature for our case (400 nm probe light, normal incidence), using the relaxation time value $\tau=2.1 \cdot 10^{14} \mathrm{~s}^{-1}=\left(\omega_{c}(T)\right)^{-1}$ measured by Li et al. [35].

\section{Acknowledgements}

This work has been supported by the LiNaBioFluid project (H2020-FETOPEN2014-2015RIA, grant 665337) of the European Commission as well as by the Spanish Ministry of Economy and Competiveness through research grant TEC2014-52642-C21-R and an FPU fellowship for M.G.-L from the by the Spanish Ministry of Education.

\section{References}

1. Ito, T. \& Okazaki, S. Pushing the limits of lithography. Nature 406, 1027-1031 (2000).

2. Deubel, M., von Freymann, G., Wegener, M., Pereira, S., Busch, K., and Soukoulis, C.M. Direct laser writing of three-dimensional photonic-crystal templates for telecommunications. Nature Mat. 3, 444-447 (2004).

3. Whitesides, G. M., Mathias, J. P., Seto, C.T. Molecular self-assembly and nanochemistry: a chemical strategy for the synthesis of nanostructures. Science 254, 1312-1319 (1991).

4. Pàmies, P. Guiding self-assembly, Nature Mat. 11, 564 (2012).

5. Birnbaum, M. Semiconductor surface damage produced by ruby lasers. J. Appl. Phys. 36, 3688-3689 (1965).

6. Temple, P. \& Soileau, M. Polarization charge model for laser-induced ripple patterns in dielectric materials. IEEE J. Quantum Electron. 17, 2067-2072 (1981).

7. van Driel, H. M., Sipe, J. E., and Young, J. F. Laser-Induced Periodic Surface Structure on Solids: A Universal Phenomenon, Phys. Rev. Lett. 49, 1955-1959 (1982).

8. Young, J. F., Preston, J. S., van Driel, H. M., and Sipe, J. E. Laser-induced periodic surface structure. II. Experiments on Ge, Si, Al, and brass. Phys. Rev. B 27, 1155-1172 (1983).

9. Bonse, J., Krüger, J., Höhm, S. \& Rosenfeld, A. Femtosecond laser-induced periodic surface structures. J. Laser Appl. 24, 042006 (2012). 
10. Öktem, B., Pavlov, I., Ilday, S., Kalaycıoglu, H., Rybak, A., Yavas, S., Erdogan, M. and Ilday, F. Ö. Nonlinear laser lithography for indefinitely large-area nanostructuring with femtosecond pulses, Nature Photon. 7 897-901 (2013).

11. Tsibidis, G. D., Fotakis, C., and Stratakis, E. From ripples to spikes: A hydrodynamical mechanism to interpret femtosecond laser-induced self-assembled structures. Phys. Rev. B 92 041405R (2015).

12. Sipe, J. E., Young, J. F., Preston, J. S., and van Driel, H. M. Phys. Rev. B 27, 1141-1154 (1983).

13. Huang, M., Zhao, F., Cheng, Y., Xu, N., and Xu, Z. Origin of laser-induced nearsubwavelength ripples: interference between surface plasmons and incident laser. ACS Nano 3, 4062-4070 (2009).

14. Bonse, J., Rosenfeld, A., and Krüger, J. On the role of surface plasmon polaritons in the formation of laser-induced periodic surface structures upon irradiation of silicon by femtosecond-laser pulses. J. Appl. Phys. 106104910 (2009).

15. Tsibidis, G. D., Barberoglou, M., Loukakos, P. A., Stratakis, E., and Fotakis, C. Dynamics of ripple formation on silicon surfaces by ultrashort laser pulses in subablation conditions. Phys. Rev. B 86115316 (2012).

16. Colombier, J. P., Garrelie, F., Faure, N., Reynaud, S., Bounhalli, M., Audouard, E., Stoian, R., and Pigeon, F. J. Appl. Phys. 111, 024902 (2012).

17. Zhang, H., Colombier, J.P., Li, Ch., Faure, N., Cheng, G., and Stoian, R. Coherence in ultrafast laser-induced periodic surface structures, Phys. Rev. B 92, 174109 (2015).

18. Höhm, S., Herzlieb, M., Rosenfeld, A., Krüger, J., Bonse, J. Dynamics of the formation of laser-induced periodic surface structures (LIPSS) upon femtosecond two-color double-pulse irradiation of metals, semiconductors, and dielectrics. Appl. Surf. Sci., published online 18 December 2015.

19. Sokolowski-Tinten, K., Barty, A., Boutet, S., Shymanovich U., Chapman, H., Bogan, M., Marchesini, S., Hau-Riege, S., Stojanovic, N., Bonse, J., Rosandi, Y., Urbassek, H. M., Tobey, R., Ehrke, H., Cavalleri, A., Düsterer, S., Redlin, H., Frank, M., Bajt, S., Schulz, J., Seibert, M., Hajdu, J. , Treusch, R., Bostedt, C., Hoener, M., Möller, T., and Phipps, C. Short-pulse laser induced transient structure formation and ablation studied with timeresolved coherent XUV-scattering. AIP Conf. Proc.1278(1), 373-379 (2010).

20. Kafka, K. R. P., Austin, D. R., Li, H., Yi, A. Y., Cheng, J., and Chowdhury, E. A. Timeresolved measurement of single pulse femtosecond laser-induced periodic surface structure formation induced by a pre-fabricated surface groove. Opt. Exp. 23, 19432-19441 (2015).

21. Jia, X X., Jia, T. Q., Peng, N. N., Feng, D. H., Zhang, S. A., and Sun, Z. R. Dynamics of femtosecond laser-induced periodic surface structures on silicon by high spatial and temporal resolution imaging. J. Appl. Phys. 115, 143102 (2014).

22. Puerto, D., Garcia-Lechuga, M., Hernandez-Rueda, J., Solis, J., and Siegel, J. Femtosecond laser-controlled self-assembly of amorphous-crystalline nanogratings in silicon, Nanotechnology 27, 265602 (2016).

23. Downer, M.C., Fork, R.L., and Shank, C. V. Femtosecond imaging of melting and evaporation at a photoexcited silicon surface. J. Opt. Soc. Am. B 2, 595-599 (1985).

24. Sokolowski-Tinten, K., Bialkowski, J., Cavaleri, A., Von Der Linde, D., Oparin, A., Meyerter-Vehn, J., and Anisimov, S.I.. Transient states of matter during short pulse laser ablation. Phys. Rev. Lett. 81, 224-227 (1998).

25. Garcia-Lechuga, M., Siegel, J., Hernandez-Rueda, J., and Solis, J. Femtosecond laser ablation of dielectric materials in the optical breakdown regime: Expansion of a transparent shell. Appl. Phys. Lett. 105, 112902 (2014).

26. Sokolowski-Tinten, K., Bialkowski, J., and von der Linde, D. Ultrafast laser-induced orderdisorder transitions in semiconductors, Phys. Rev. B. 51, 14186-14198 (1995).

27. Sokolowski-Tinten, and von der Linde, D. Generation of dense electron-hole plasmas in silicon, Phys. Rev. B. 61, 2643-2650 (2000). 
28. Jellison Jr., G. E. and Lowndes, D. H. Measurements of the optical properties of liquid silicon and germanium using nanosecond time-resolved ellipsometry. Appl. Phys. Lett. 51, 352-354 (1987).

29. Shank, C.V., Yen, R., and Hirlimann, C. Phys. Rev. Lett. 50, 454-457 (1983).

30. Gusakov, G. M., Komarnitskii, A. A., and Em, A. S. Dynamics of optical parameters of silicon and germanium single crystals during two-pulsed nanosecond laser irradiation. physica status solidi (a) 107, 261-271 (1988).

31. Boneberg, J., Yavas, O., Mierswa, B., and Leiderer, P. Optical reflectivity of Si above the melting point. physica status solidi (b) 174, 295-300 (1992).

32. Thompson, M. O., Mayer, J. W., Cullis, A. G., Webber, H. C., Chew, N. G., Poate, J. M., and Jacobson, D. C. Silicon melt, regrowth, and amorphization velocities during pulsed laser irradiation, Phys. Rev. Lett. 50, 896-899 (1983).

33. Chaoui, N., Siegel, J., Solis, J., Afonso, C. N. Reflectivity of crystalline Ge and Si at the melting temperature measured in real time with sub-nanosecond temporal resolution, $J$. Appl. Phys. 89, 3763-3767 (2001).

34. Siegel, J., and Solis, J. Imaging of Plasma Dynamics for Controlled Micromachining. Chapter 2 in "Femtosecond Laser Micromachining: Photonic and Microfluidic Devices in Transparent Materials", Eds: Osellame, R., Cerullo, G., Ramponi, R. Series: Topics in Applied Physics, Vol. 123, Springer-Verlag Berlin Heidelberg (2012).

35. Li, K. D., and Fauchet, P. M. Drude parameters of liquid silicon at the melting temperature, Appl. Phys. Lett. 51, 1747-1749 (1987). 